\title{
What should I tell my significant other?
}

\author{
JA BARROWMAN, MB, ChB, MRCP(Lond), PHD, FRCP, FACP, FRCPC
}

W HEN YOUR DOCTOR GIVES YOU a diagnosis of one or other of the inflammatory bowel diseases (IBD), Crohn's disease or ulcerative colitis, you will, like most patients, experience mixed emotions. Often the immediate feeling is one of relief on having a definite diagnosis. This is not surprising; the fear of the unknown generated by the suspicion that one has a serious bowel disease creates a great deal of emotional tension. You may have been wondering why your doctor needed so much time and so many tests to come to the diagnosis. As you get to know more about your disease the reasons for this become clear. There is no simple urine or blood test for these diseases and their diagnosis often is quite difficult. There is only a limited number of ways in which a sick bowel can intimate that all is not well. Abdominal pain, diarrhea, constipation and bleeding are the symptoms that tell us the bowel is unhealthy, but they do not tell us which of the various diseases which can affect the bowel is causing the problem. With Crohn's disease, the symptoms may be general and nonspecific - tiredness, weight loss or lack of 'joie de vivre'. Intestinal symptoms may be minor.

In view of this, and the fact that bowel diseases are common, our imaginations run riot until a diagnosis is established. Most of us know of people with 'colitis', diverticulosis of the colon, polyps or cancers of the bowel, and anxiety mounts as we compare symptoms. So when your doctor explains the diagnosis, how it was arrived at, the general outlook and the outlook for you as an individual with your particular variety of the condition, you will probably experience some relief from the tensions leading up to this point.

It is characteristic of IBD, in particular Crohn's disease, that the diagnosis often is not reached for several months or even years, and many patients will look back on that period of their lives with mixed feelings.

Your doctor will probably explain the disease, ulcerative colitis or Crohn's disease, to you during one or two encounters. These are chronic conditions of idiopathic (unknown) cause. Doctors are well aware of the fact that patients do not take in all the information about a diagnosis and its implications in one brief office visit; you will be thinking about so many things at once - the future, how this disease will affect your life, family, work, leisure. So do not be reticent in requesting a further appointment to discuss the problem. It is very important that your doctor explain the extent of your particular disease. Ulcerative colitis, for example, can be exten- sive, involving the entire colon or may present as a milder condition, ulcerative proctitis, which is confined to the rectum and generally has a much better outlook. Similarly, Crohn's disease may be confined to one small segment of the bowel or may be more extensive. Again your doctor will try to give you a realistic outlook based on knowledge of your particular problem.

When your doctor has made the diagnosis, he or she will provide you with literature on various aspects of the disease. If not, you must ask for it. The Canadian Foundation for Ileitis and Colitis (CFIC) has produced excellent pamphlets on the subject which address the issues, and the literature constantly is being updated. You will have questions which are peculiar to you, your family and your lifestyle. You must write them down and address them to your doctor at a subsequent interview. You may wish to have a close relative or friend attend the interview and if you are a youngster, your parents should attend.

But you cannot live in a doctor's office. You will need to talk to your family, fellow workers and employer, school teachers and others who play a big part in your life.

You must be frank in explaining the problem as best you can to those people who need to know. Nothing except misunderstanding is served by concealing things from family, friends, employer or teachers. The pamphlets 
(produced as a service to patients by the CFIC) deal with questions related to the nature of the diseases and the outlook of patients with the conditions. Medical, surgical and psychological aspects are discussed, as well as special questions related to sex, pregnancy, heredity and matters of concern to certain age groups, such as teens. These pamphlets are freely available and you should offer copies to anyone whom you feel needs the information.

When you wonder how to tell other people about your disease, it helps to put yourself in their shoes for a moment. Yourhusband/wife, boyfriend/girlfriend will want to know (but be unsure of how to ask you) what the long term implications are. Is there a lifetime of chronic ill health ahead? Is surgery likely or even inevitable? Will there be a need for extensive medical insurance and will it cost a great deal? Is the disease contagious? Is it possible to precipitate or avoid relapses by some lifestyle behaviour? Will you be able to travel? What recreational pursuits are possible or inadvisable? Can one have a normal sex life, pregnancies and children? Is the disease passed down the generations? Is there a cancer risk? Apart from cancer, what serious complications of the disease can arise in the future? What are the treatment options? Are the drugs that are used safe? Will they be expensive?

To all these various questions, it is possible to give a reassuring answer. Yes, the diseases are chronic and of unknown cause, waxing and waning in severity. For the majority of patients, however, life usually is full and satisfying, but it is wise to be realistic. Some patients experience frequent relapses needing medical or surgical intervention. It might help to consider other chronic diseases such as diabetes, arthritis and asthma as belonging to a similar category.

Surgery is certainly an option in both diseases but it is by no means a first line of treatment. In the case of ulcerative colitis, the disease can occasionally be so severe and aggressive that total removal of the large bowel (colon) is necessary. In this case, since ulcerative colit is affects only the colon and not the small intestine, the patient will no longer have the disease after this surgical procedure. Surgery frequently is carried out in Crohn's disease for some complications, often obstruction of the bowel or an abscess in the abdomen. It is important to recognize that unlike a total colectomy (surgical removal of the large intestine) for ulcerative colitis, removal of a segment of the large or small intestine for Crohn's disease does not guarantee a cure. Unfortunately a new section of the bowel can be affected by Crohn's disease at a later date.

\section{MEDICAL INSURANCE}

Medical insurance needs are a decision for you as an individual and depend on personal factors. However, anticipating the possibility of periods of disability it may be prudent to take out some such insurance. You will, like everyone else, also probably wish to take out life insurance to provide for your dependents. Unfortunately, it is probable that your insurance will be difficult to obtain or will cost you more than someone without these diseases and you may need to 'shop around' or ask advice from another person to get the best possible deal. After you make application for insurance, your physician will be approached by the insurance company for information about your particular case; the medical advisor of the insurance company will assess the situation and advise the company about the terms of your policy. You should feel free to discuss this matter with your physician who will do whatever is possible to assist you.

Idiopathic IBD are not contagious and they cannot be passed to family or associates. In fact, if they were contagious they would not be idiopathic and their cause and cure would have been found by now.

Little is known about precipitating factors in IBD. In general terms, one should avoid extreme physical stress, eat a nutritious diet, and get regular exercise and rest. We suspect that some relapses may be precipitated by intestinal or other infections but there is little one can do to avoid this. It is possible that some antibiotics given, for example for respiratory infections, can in some way precipitate an attack. It is also possible that periods of emotional stress may lead to relapses but it often is difficult to separate 'cart and horse' in this situation.

\section{TRAVEL}

Patients with IBD should not be alarmed by the prospects of travel either at home or abroad. The diseases are well-recognized worldwide and physicians are familiar with their management. However, in certain countries such as India, Crohn's disease is very uncommon. In countries where sanitation is poor there is a greater chance of intestinal infections which may precipitate a relapse. You should take care to avoid such infections, for example by drinking only bottled water and eating well-cooked meat and fish. It is best to avoid vegetables and fruits which you have not prepared yourself. It is essential to take additional medical insurance for foreign travel and to take your drug supplies with you in welllabelled containers; it is prudent to take a letter from your doctor explaining the need for these medications.

If there is a likelihood that you will require medical attention in another part of Canada, United States or overseas, a letter from your doctor outlining the major aspects of your disease and its treatment will be useful. You may wish to wear a medical bracelet; you can apply for this from the Canadian Medical Alert Foundation, Inc, 293 Eglinton Avenue East, Toronto, Ontario, M4P $2 Z 8$ (telephone 1-800-668-1507).

\section{ACTIVITIES}

Patients suffering from IBD can take part in all forms of recreational activity including the most vigorous sports. It may be that if your disease is active you may tire rather easily but you must use your judgement as to how much you take on. Since there is no bar to a full leisure life, it is up to you to make the most of it.

There are several questions you will want to discuss with your partner regarding your sex life. In general, patients with IBD can have a normal sex life. This also applies to patients who have an ileostomy (an opening of 
the small bowel on to the abdominal wall following surgical removal of the large intestine). If the disease is active, you may lose your sex drive for some time, but this will resume as your condition settles. You can have children; patients with ulcerative colitis seem to be normally fertile but both men and women with active Crohn's disease may be relatively less fertile. In general, pregnancies proceed normally in patients with ileitis or colitis and conventional drug treatment of the disease during pregnancy does not affect the baby. Pregnancy itself does not alter the chances of relapse of the diseases.

A very important question you will ask relates to the chance of your children having the disease. We know that IBD are more common in the relatives of any patient with ulcerative colitis or Crohn's disease than in the general population. Occasionally a patient with one disease may have a relative with the other disease, but for the most part it is the same disease in close relatives. So there is some risk of your children having the disease. The estimates are that if you have ulcerative colitis, your children have only a one in 10 chance of having the disease; for Crohn's disease, the likelihood is a little higher, two to three chances in 10. You must judge for yourself, but for most people the risk is not seen as high enough to avoid pregnancy.

\section{COMPLICATIONS}

What about complications of IBD? There is a cancer risk with both diseases (ulcerative colitis more than Crohn's disease). This is not a major risk and usually applies only to patients who have had the disease for more than 10 years. Generally, there is more risk if the disease has been frequently active and involves the whole of the large intestine. Your doctor will discuss this risk with you and will initiate surveillance, with periodic $\mathrm{x}$-rays and colonoscopy, where appropriate. Given the fact that doctors are very familiar with this problem, you can have confidence that appropriate steps will be taken to safeguard you against this risk.

There are other complications which you should know about. For ul- cerative colitis, severe disease may not be controllable with medical treatment and it may be necessary to remove the large bowel surgically. The bowel can become paralyzed and distended, with the risk of perforation. This is called toxic megacolon and is uncommon, but is an emergency situation requiring prompt surgery. Crohn's disease patients can also develop abscesses or fistulae (abnormal communications between the bowel and other segments of the bowel, bladder, vagina or skin); narrowed segments of the bowel may lead to obstruction. Most of these problems require surgery. Finally, although the diseases primarily affect the large and/or small intestine, associated problems can arise in the skin (various types of rash or ulcers), the mouth (painful shallow ulcers), the joints (swollen painful joints such as the knees or arthritis of the spine, particularly its lower part) and the eyes (inflammation with painful red eyes). These can all be treated and controlled medically; there is a good chance that you may never have any of these nonintestinal problems. Finally, in Crohn's disease, chronic inflammation quite often affects the region around the anus, with fissures that are painful particularly when defecating.

\section{TREATMENT PLAN}

Once your condition has been diagnosed, your doctor will outline your treatment plan. This is very important and has implications for various aspects of your daily life. Treatment is primarily medical and surgical. Surgical treatment has already been referred to. Medical treatment, besides drugs, includes dietary measures and, in some instances, psychological counselling. As your disease is a lifelong one, it is wise to enquire about a 'drug plan' to cover medication costs. While some of your medications are quite cheap, certain preparations, particularly drugs, given in the form of enemas, are very costly. You will also want to know about the unwanted effects of your drugs especially since you may need to take these drugs for long periods of time.

Excellent CFIC pamphlets, Drug Therapy of IBD and Sex and Pregnancy with IBD give detailed answers to these questions and it is very important that you read these carefully. Your doctor will explain any areas that you may find confusing.

The main specific drugs used in treating IBD are steroids given orally, by enema, or occasionally intravenously. They are powerful suppressors of inflammation. They are generally used to control acute attacks and because of a variety of unwanted effects, doctors will tend to use them in short courses, or, if long courses are necessary, in low doses.

Sulphasalazine (Salazopyrine; Pharmacia) is used in Crohn's colitis but its most important use is as a means of preventing or reducing the frequency of relapses in ulcerative colitis. For this reason, it usually is taken on a long term basis. It, too, has unwanted effects which generally show up early in treatment. Once you have settled down on sulphasalazine, the unwanted effects are few.

5-aminosalicylate (5-ASA) is a derivative of sulphasalazine which is relatively new. It is likely to be used for the same purposes as sulphasalazine. It appears to have considerably fewer unwanted effects than sulphasalazine, and those that do occur are usually minor.

Occasionally, your doctor may use azathioprine or 6-mercaptopurine if you have Crohn's disease. These are powerful suppresssors of immune reactions. They can have important unwanted effects and will only be given to you under careful medical supervision. The antibiotic, metronidazole (Flagyl; RhônePoulenc), is used in Crohn's colitis and in patients with Crohn's anal and perianal disease.

A variety of drugs with nonspecific effects are used to control symptoms in IBD: antidiarrheal agents include: codeine, diphenoxylate (Lomotil; Searle) and loperamide (Imodium; Janssen). Bulk forming agents such as psyllium (Metamucil; Procter \& Gamble) are used sometimes as antidiarrheal agents, and in other settings, as laxatives giving a soft bulky stool. A special compound, cholestyramine (Questran; Bristol), binds the body's natural laxatives, bile salts, and is useful in treating diarrhea in patients with Crohn's ileitis and in 
patients who have had the lower small intestine removed surgically. Compared with the specific drugs listed in previous paragraphs, the nonspecific drugs generally have few unwanted effects.

In severe nutritional deficiency, especially in extensive Crohn's disease, your doctor may need to admit you to hospital for intensive medical treatment. In this case, the use of 'elemental' diets consisting of simple readily assimilable foodstuffs or intravenous feeding may be necessary.

Finally, mention should be made of surgical treatment. It is important to know that a colectomy does not necessarily mean that you will have an ileostomy. Newer surgical procedures are available in which the small intestine can be joined to the rectum or even the anal canal. Your surgeon will discuss the pros and cons of such surgery with you before embarking on the operation.

Fortunately, medical treatment in combination with careful supervision controls the disease in a majority of patients. Although the specific drugs do have certain unwanted effects, these do not occur in every patient, are for the most part reversible on discontinuing the drug and are often minor in nature. Your doctor will adjust the choice and dose of drugs in your particular case as necessary. New medications are constantly being explored and your doctor may ask you to consider taking part in a trial of a new agent. He will explain the nature of the study to you before asking for your consent in taking part; of course there is no obligation to you to do so.

It is essential for parents whose child has IBD to speak to their teachers to explain the ways in which the child is affected, so the teachers can understand the child's pain, and the urgent and sometimes frequent visits to the washroom. You may wish to ask the doctor to answer any specific queries your child's teachers may have. The teacher who understands the reality of the child's problems can respond in a supportive and matter-of-fact way, neither minimizing nor dramatizing the difficulties. The other children, and your child too, will follow the example of an understanding teacher who appreciates the child's needs without being overly protective. You can assure the teacher that IBD are not at all contagious. It is probably wise to keep a change of clothing at school. There may be occasions when the child is in pain or discomfort and will not feel like physical education or other energetic activities. Beyond normal encouragement, the child should not be forced to participate, but should be allowed to judge for him/herself. Physical exercise will not do any harm and, indeed, will probably help. If medications are prescribed they can be taken at home; seldom is it necessary to take medication during school hours.

Regarding your employment, it should first be emphasized that you are under no legal obligation to discuss personal medical details with your employer. That said, however, it is in everyone's interests not to conceal important information that assists you and your employer to achieve a productive relationship. When you are being interviewed for a position, it is wise to be frank and explain the nature of the problem. Your potential employer might have questions about your future similar to some of those already discussed. Again, the CFIC literature is helpful. Some employers may require a pre-employment medical examination. One must realize that the health requirements for some jobs do not allow the employment of people with a variety of chronic diseases. You would not wish your airline pilot to suffer from serious heart disease or have visual problems! It is wise to enquire ahead of time about medical requirements for the various jobs/professions you are considering. Patients with IBD are happily employed in a wide variety of jobs. Like teachers and school children, employers should be made aware of the practical problems you are likely to face, ie, days when you feel substandard, periods when pain tends to distract you and, always, the need for easy access to a washroom. An understanding employer to whom you have confided your problem can help make your working life proceed smoothly.

PATIENT INFORMATION: Pamphlets published by the Canadian Foundation for Ileitis and Colitis: The Pain is Real, Psychological Aspects of IBD, Questions about Crohn's Disease and Ulcerative Colitis, More Questions about Crohn's Disease and Ulcerative Colitis, Drug Therapy of IBD, Sex and Pregnancy with IBD, A Teacher's Guide to Crohn's Disease and Ulcerative Colitis and Information for Teenagers about Crohn's Disease and Ulcerative Colitis. For further information write to: The Canadian Foundation for Ileitis and Colitis, 21 St Clair Avenue East, Suite 301, Toronto, Ontario M4T 1L9, telephone (416) 920-5035. 


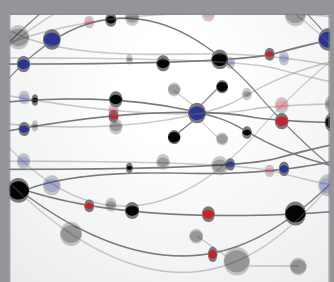

The Scientific World Journal
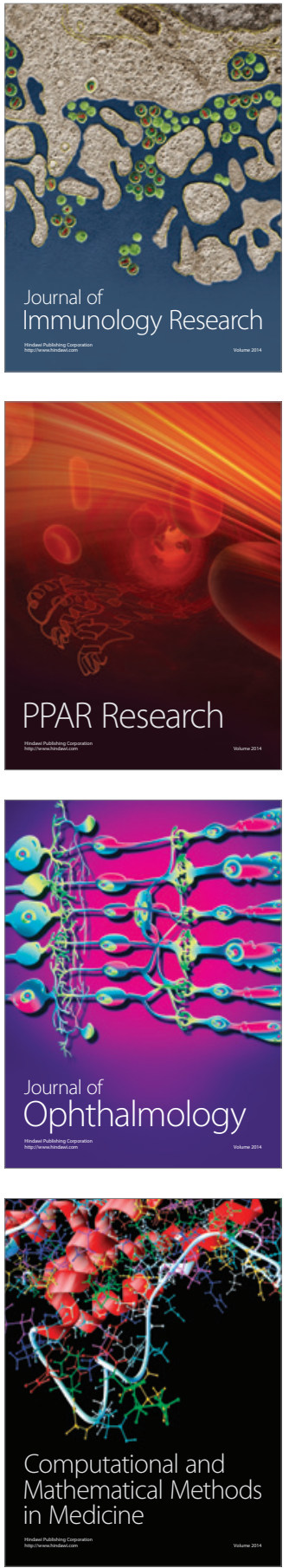

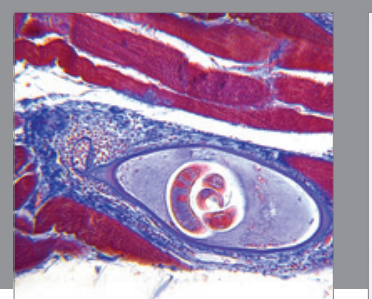

Gastroenterology Research and Practice

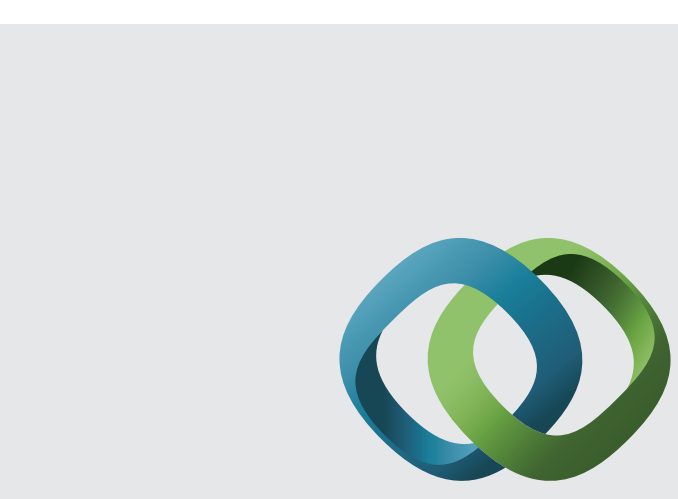

\section{Hindawi}

Submit your manuscripts at

http://www.hindawi.com
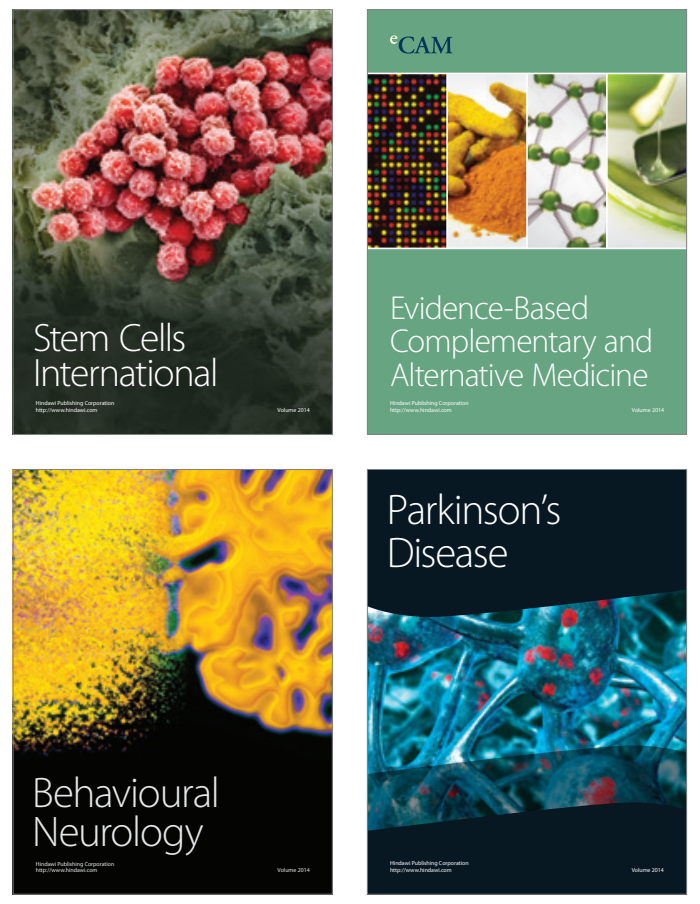
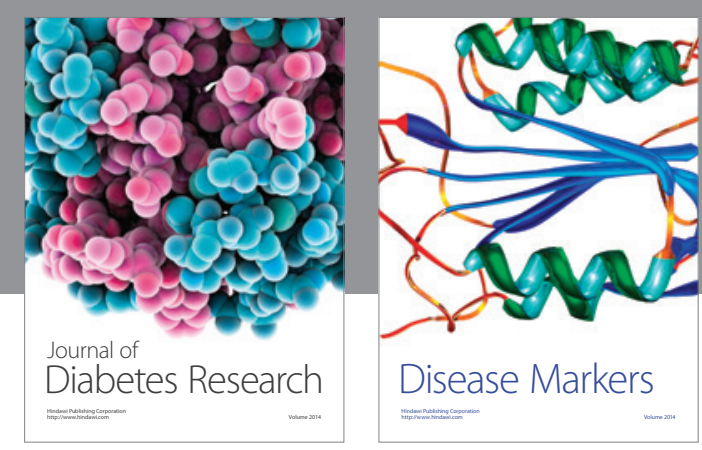

Disease Markers
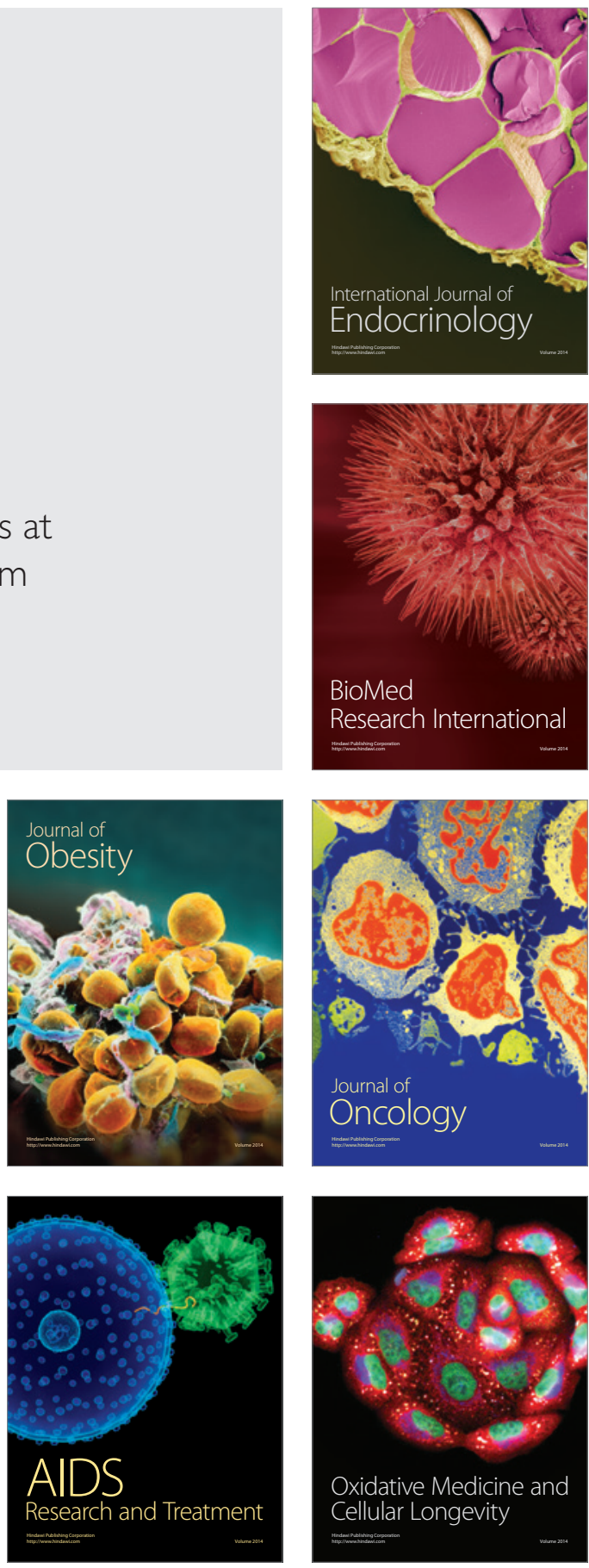